\title{
Anticorps catalytiques ou abzymes
}

Sébastien Lacroix-Desmazes, Jagadeesh Bayry, Michel D. Kazatchkine, Srini V. Kaveri

$>$ Le concept selon lequel certains anticorps pourraient posséder une activité enzymatique a été énoncé pour la première fois par Linus Pauling en 1942. Selon L. Pauling, si la structure des sites de liaison à l'antigène des anticorps était produite de manière aléatoire, on devrait trouver des anticorps dont les sites de liaison ressemblent aux sites actifs d'enzymes et qui pourraient être dotés d'une activité enzymatique. II fallut attendre les travaux de G. Köhler et C. Milstein en 1975 [1], permettant la production des anticorps monoclonaux, pour confirmer cette hypothèse: les premiers anticorps catalytiques, ou «abzymes » (antibody-enzyme), furent produits en 1986. Les différentes approches qui permettent d'obtenir des «abzymes » sont présentées ci-dessous, et l'importance de l'activité catalytique des anticorps en pathologie humaine est discutée.

\section{Obtention d'《 abzymes 》}

par immunisation à l'aide d'haptènes

La grande majorité des «abzymes » a été produite par immunisation à l'aide d'haptènes qui sont des analogues stables d'états de transition de réactions chimiques. En 1986, A. Tramontano a produit des anticorps monoclonaux hydrolysant les liaisons ester en utilisant comme haptène une molécule analogue de l'état de transition de l'hydrolyse des esters carboxyliques (Figure 1) [2]. La

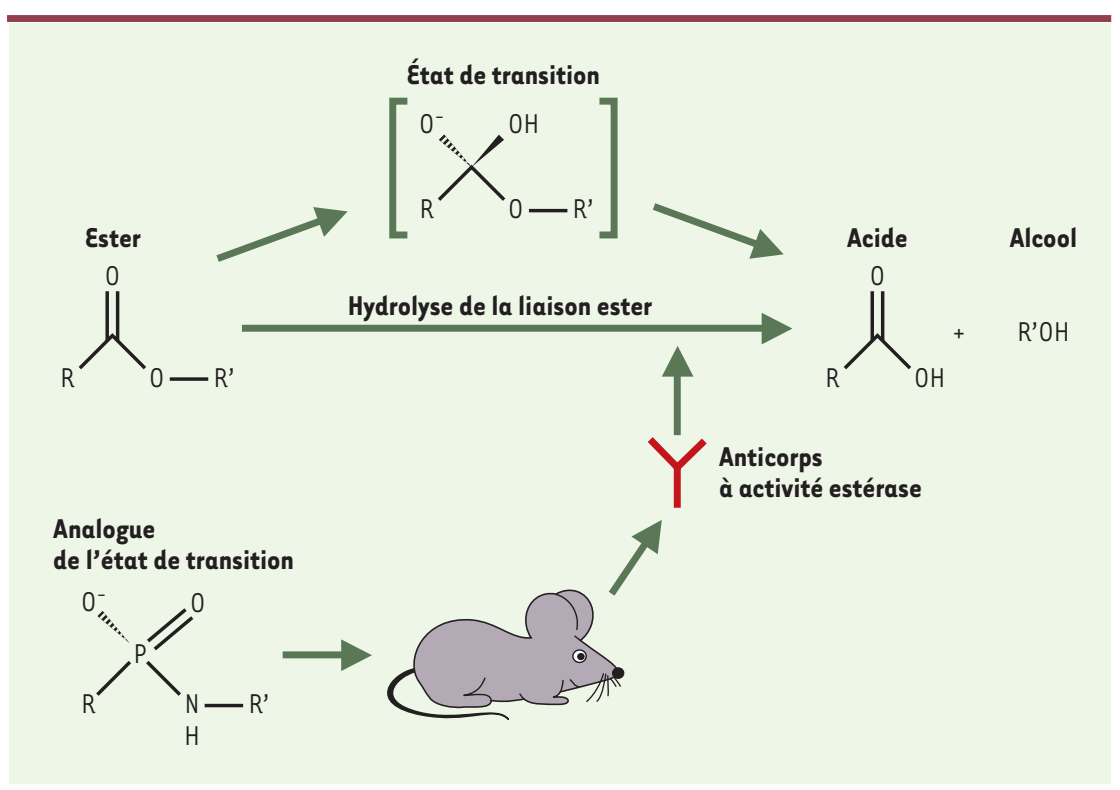

Figure 1. Hydrolyse de la liaison ester par des anticorps produits après immunisation à l'aide d'un haptène analogue de l'état de transition de la réaction (d'après [28]).
Inserm U.430,

Institut des Cordeliers,

15 , rue de l'École de Médecine,

75270 Paris Cedex 06,

France.

sebastien.lacroix-desmazes

$@$ @430.bhdc.jussieu.fr

même année, S.J. Pollack a produit un anticorps monoclonal murin de forte affinité pour l'analogue d'état de transition p-nitrophénylphosphorylcholine. Cet anticorps catalysait l'hydrolyse de la liaison carbonate [3]. Deux approches alternatives ont depuis été développées: l'immunisation réactive se fonde sur l'utilisation d'haptènes si fortement réactifs qu'une réaction chimique survient avec le site de liaison de l'anticorps pendant l'immunisation [4]; la technique du bait and switch utilise des haptènes qui comportent des structures complémentaires (par exemple, des charges électriques) aux résidus désirés dans le site de liaison de l'«abzyme » [5]. Ces stratégies, qui dépendent essentiellement de la synthèse chimique d'haptènes, ont permis la production d'anticorps catalysant des réactions telles que les réactions d'addition électrophile-élimination, de racémisation, d'isomérisation, d'hydrolyse et de formation de liaisons carbone-carbone [6, 7]. Un des intérêts majeurs de l'utilisation d'analogues d'états de transition est la possibilité de produire des «abzymes » qui catalysent des réactions thermodynamiquement ou cinétiquement défavorables, qui ne sont pas catalysables par les enzymes naturelles connues ou par les méthodes chimiques disponibles [8].

\section{Obtention d'《 abzymes 》 par} manipulation du réseau idiotypique Une approche plus biologique utilisée avec succès fait intervenir la notion d'« image interne » constitutive du 
réseau idiotypique, initialement proposée par Niels Jerne en 1974 [9]. Des animaux sont immunisés à l'aide d'une enzyme avec pour objectif la production d'un anticorps monoclonal (Abl) dont le site de liaison à l'antigène possède des structures complémentaires à celles du site actif de l'enzyme (Figure 2). Des anticorps monoclonaux (Ab2) dirigés contre le site de liaison à l'antigène de l'Abl, sont alors produits. Certains des Ab2 portent une image du site actif de l'enzyme et miment la fonction catalytique de cette enzyme. Cette approche a permis la production d'anticorps doués d'une activité estérase [10], amidase [11] ou sérine protéase [12], en utilisant respectivement l'acétylcholinestérase, la $\beta$-lactamase et la subtilisine comme immunogènes. Les paramètres cinétiques des anticorps catalytiques sont la Vmax (vitesse de catalyse maximale à concentration saturante en substrat), le Km (affinité pour le substrat), le Kcat (nombre de fois où la catalyse a lieu par unité de temps) et l'efficacité catalytique (exprimée en nombre de moles de substrat catalysées par mole d'enzyme et par unité de temps). Les paramètres cinétiques des « abzymes » anti-idiotypiques sont plus faibles que ceux des enzymes d'origine, mais sont toutefois plus élevés que ceux d'《abzymes » de même activité produits par les analogues d'états de transition.

\section{Anticorps catalytiques chez l'homme}

Des «abzymes » sont produits de manière «naturelle » par le système immunitaire, en l'absence d'immunisation délibérée. II a été proposé que ces anticorps catalytiques naturels pourraient participer directement à l'élimination des débris qu'engendre le métabolisme de l'organisme dans les conditions physiologiques [13]. La présence dans le lait humain normal d'anticorps catalytiques dotés d'une activité protéine kinase et d'anticorps à activité désoxyribonucléase suggère un rôle protecteur des «abzymes » dans les conditions physiologiques [14, 15].
Toutefois, la majorité des études faites chez l'homme démontre que la prévalence des «abzymes » augmente dans les conditions pathologiques, et en particulier lors de maladies auto-immunes. Les premiers «abzymes » décrits chez I'homme ont été isolés chez des patients asthmatiques et clivaient le peptide intestinal vasoactif (VIP) [16]. Depuis, des anticorps protéolytiques contre la thyroglobuline et la prothrombine ont été décrits respectivement chez des patients atteints de thyroïdite ou de myélome multiple $[17,18]$. Nous avons mis en évidence la présence d'anticorps hydrolysant le facteur VIII (FVIII) chez certains patients hémophiles $A$ ayant développé des anticorps anti-FVIII inhibiteurs après administration de FVIII pour corriger le déficit en FVIII [19]. Des anticorps hydrolysant I'ADN et I'ARN ont été isolés à partir du sérum de patients atteints de différentes maladies auto-immunes systémiques: lupus érythémateux disséminé, sclérodermie, polyarthrite rhumatoïde ou sclérose en plaques [20-22]. Il est en général difficile de déterminer si les «abzymes » détectés chez les patients jouent un rôle dans l'étiologie de la maladie, et si leur apparition résulte de la maladie ou est à l'origine des manifestations cliniques. Cependant, dans le cas des patients hémophiles porteurs d'anticorps anti-FVIII inhibiteurs, I'hydrolyse du FVIII par les anticorps protéolytiques pourrait participer directement à l'inactivation du FVIII et contribuer à aggraver le déficit de l'hémostase [23].

\section{Activité bactéricide des anticorps}

P. Wentworth et al. ont récemment démontré que tous les anticorps sont potentiellement dotés d'activité bactéricide [24]. Les anticorps sont en effet intrinsèquement capables de favoriser la production de peroxyde d'hydrogène et d'ozone. Cette propriété est conservée entre les anticorps de différentes espèces, elle est indépendante de leur spécificité antigénique ou de leur composition en chaînes lourdes et légères, mais est partagée par d'autres molécules telles que l'ovalbumine de poulet et la $\beta$-galactosidase [24]. Dans le cas des anticorps, les travaux suggèrent un rôle prépondérant des résidus Trp enfouis dans la molécule dans le processus d'oxydation de l'eau, et en particulier

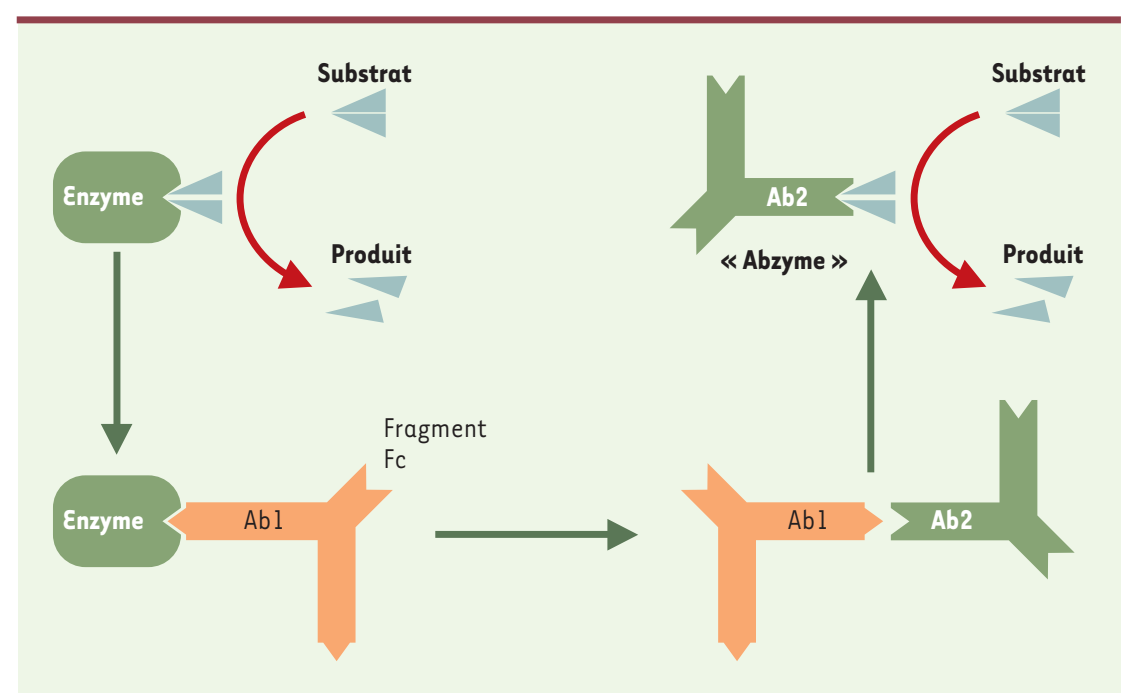

Figure 2. Production d'《 abzymes 》 par manipulation du réseau idiotypique. Un organisme est immunisé à l'aide d'une enzyme. Les anticorps monoclonaux produits (Abl) qui neutralisent l'activité enzymatique sont utilisés pour induire des anticorps anti-idiotypiques (Ab2). Certains Ab2, appelés anticorps catalytiques ou «abzymes », présentent un site de liaison à l'antigène qui ressemble structurellement au site actif de l'enzyme et sont dotés d'activité enzymatique. 
l'implication des résidus Trp-36 et Trp47 qui sont conservés dans plus de $99 \%$ des anticorps [25]. À ce titre, l'activité d'oxydation de l'eau des anticorps ne permet pas de les assimiler à des «abzymes » dans la mesure où cette activité n'est pas dépendante de la nature des gènes qui codent pour les régions variables des anticorps et n'est pas associée à une spécificité particulière des anticorps pour un substrat/ antigène donné. Cette observation étaye cependant l'hypothèse d'un rôle protecteur intrinsèque des anticorps dans les conditions physiologiques, rôle indépendant de leur capacité de neutraliser les antigènes étrangers, de faciliter leur endocytose par les cellules présentatrices de l'antigène et de permettre leur élimination. Toutefois, la production $d^{\prime} \mathrm{H}_{2} \mathrm{O}_{2}$ qu'induisent les IgG anti-GPIIla (glycoprotéine plaquettaire équivalente de la chaîne $\beta 3$ des intégrines et qui s'associe à la GPIIb pour former un complexe essentiel à l'activation plaquettaire), qui surviennent au cours de l'infection par le VIH, a été incriminée dans l'induction par ces anticorps d'une thrombocytopénie [26].

\section{Conclusions}

Deux catégories d'anticorps catalytiques peuvent être distinguées (Figure 3) $(\rightarrow)$. (1) Les « abzymes induits » n'apparaissent qu'après immunisation active à l'aide de mimes moléculaires: l'immunisation par des haptènes analogues d'états de transitions ou par des structures complémentaires de sites actifs d'enzymes (régions variables d'Abl) permettrait de puiser, au sein du répertoire disponible des anticorps, des réactivités susceptibles d'acquérir une activité catalytique au cours du processus de maturation d'affinité. (2) Les «abzymes naturels », en revanche, sont produits par le système immunitaire de manière constitutive en conditions physiologiques: le potentiel catalytique des anticorps serait inscrit dans le répertoire des gènes codant pour leurs régions variables et pourrait être hérité de mécanismes primitifs de défense de l'organisme contre les agents pathogènes. La production d'«abzymes » augmente dans le cas de déséquilibres du système immunitaire, lors de la grossesse ou de désordres auto-immuns par exemple. La fréquence des clones catalytiques est aussi accrue chez les souris NZB/W, MRLIpr/lpr ou SJL/J qui sont des modèles expérimentaux classiques de maladies autoimmunes [27]. Or, I'apparition de manifestations autoimmunes ressemblant au lupus chez les souris MRL-Ipr/Ipr est associée à un défaut de la voie d'induction d'apoptose dépendante de Fas et à la persistance à la périphérie de lymphocytes autoréactifs délétères pour l'organisme. Ces observations suggèrent que les lymphocytes B exprimant des anticorps catalytiques «naturels » sont soumis à une forte régulation négative dans les conditions physiologiques. $\diamond$

Catalytic activity of antibodies

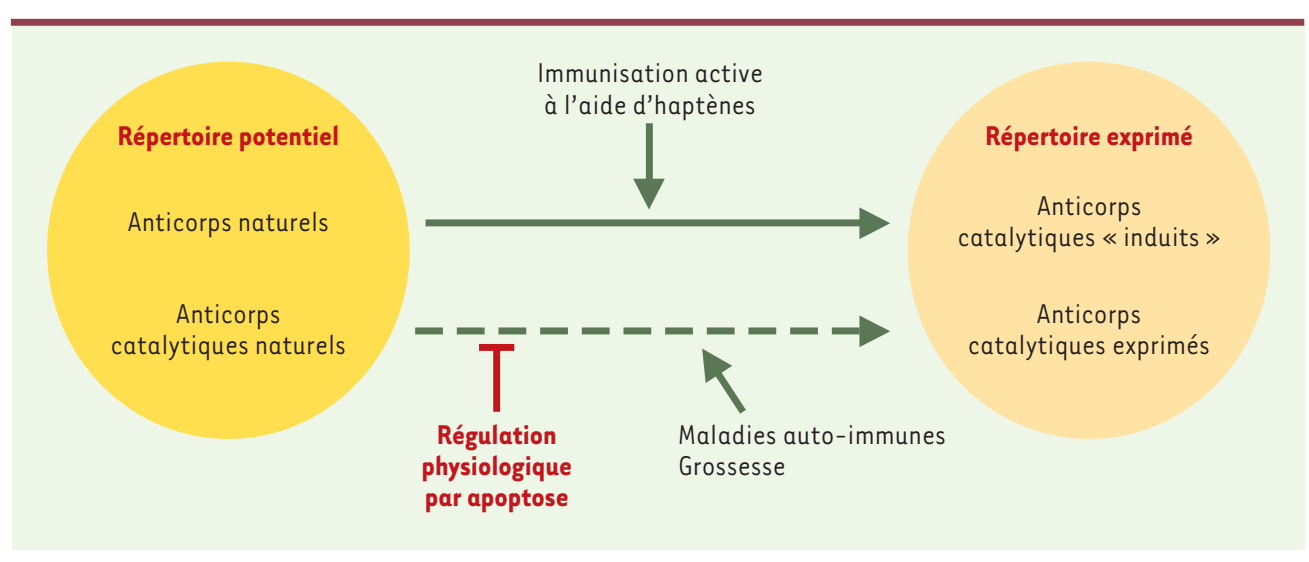

Figure 3. Répertoire des anticorps potentiellement produits par l'organisme. Le répertoire est déterminé par le nombre des gènes $V H, D H, J H, V L$ et $J L$ qui codent pour les régions variables $(V)$ des immunoglobulines et par la diversité jonctionnelle (J) qui survient au cours du réarrangement somatique. Ce répertoire potentiel comprend des anticorps dont les sites de liaison à l'antigène peuvent reconnaître l'ensemble des antigènes étrangers ou autologues (anticorps naturels), ou peuvent ressembler structurellement au site actif d'enzymes et posséder une activité catalytique (anticorps catalytiques naturels). Dans les conditions physiologiques, les clones de lymphocytes B produisant des anticorps naturellement dotés d'activité catalytique sont soumis à une régulation négative par apoptose. Les anticorps catalytiques ne sont exprimés qu'après immunisation active à l'aide d'haptènes analogues d'états de transition, ou si les mécanismes physiologiques de régulation sont perturbés, lors de grossesses ou de maladies auto-immunes par exemple.

\section{RÉFÉRENCES}

1. Köhler G, Milstein C. Continuous culture of fused cells secreting antibody of predefined specificity. Nature 1975; 256 : 495-7.

2. Tramontano A, Janda KD, Lerner RA. Catalytic antibodies. Science 1986; 234: 1566.

3. Pollack SJ, Jacobs JW, Schultz PG. Selective chemical catalysis by an antibody. Science 1986; 234: 1570.

4. Wirsching P, Ashley JA, Lo $\mathrm{CHL}$, et al. Reactive immunization. Science 1995; 270 : 1775-8.

5. Wentworth $P$, Liu YQ, Wentworth $A D$, et al. A bait and switch hapten strategy generates catalytic antibodies for phosphodiester hydrolysis. Proc Natl Acad Sci USA 1998; 95 : 5971-5.

6. Flanagan $M \varepsilon$, Jacobsen JR, Sweet $\varepsilon$, Schultz PG. Antibody-catalyzed retroaldol reaction. J Am Chem Soc 1996; 118: 6078-9.

7. Hasserodt J. Organic synthesis supported by antibody catalytis. Synlett 1999; 12: 2007-22. 
8. Hilvert D. Critical analysis of antibody catalysis. Annu Rev Biochem 2000; 69: 751.

9. Jerne NK. Toward a network theory of the immune system. Ann Immunol (Paris) 1974; 125C: 373-89.

10. Izadyar L, Friboulet A, Remy $\mathrm{MH}$, et al. Monoclonal antiidiotypic antibodies as functional internal images of enzyme active sites: production of a catalytic antibody with a cholinesterase activity. Proc Natl Acad Sci USA 1993; 90: 8876-80.

11. Avalle B, Thomas D, Friboulet A. Functional mimicry: elicitation of a monoclonal anti-idiotypic antibody hydrolizing betalactams. FASEB J 1998; 12 : 1055-60.

12. Pillet D, Paon M, Vorobiev II, et al. Idiotypic network mimicry and antibody catalysis: lessons for the elicitation of efficient anti-idiotypic protease antibodies. I Immunol Meth 2003; 269: 5-12.

13. Friboulet A, Avalle B, Debat $H$, Thomas D. A possible role of catalytic antibodies in metabolism. Immunol Today 1999; 20 : 474-5.

14. Kit Yy, Semenov DV, Nevinsky GA.

Phosphorylation of different human milk proteins by human catalytic secretory immunoglobulin A. Biochem Mol Biol Int 1996; 39: 521-7.

15. Kanyshkova TG, Semenov DV, Khlimankov D, et al. DNA-hydrolyzing activity of the light chain of IgG antibodies from milk of healthy human mothers. FEBS Lett 1997; 416: 23-6.

16. Paul S, Volle DJ, Beach CM, et al. Catalytic hydrolysis of vasoactive intestinal peptide by human autoantibody. Science 1989; 244 : 1158-62.

17. Li L, Paul S, Tyutyulkova S, et al. Catalytic activity of anti-thyroglobulin antibodies. J Immunol 1995; 154: 3328-32.

18. Thiagarajan $P$, Dannenbring $\mathrm{R}$, Matssura K, et al. Monoclonal antibody light chain with prothrombinase activity. Biochemistry 2000; 39: 6459-65.

19. Lacroix-Desmazes $S$,
Moreau A, Sooryanarayana $S$, et al. Catalytic activity of antibodies against factor VIII in patients with hemophilia A. Nat Med 1999; 5 : 1044-7.

20. Shuster AM, Gololobov GV, Kvashuk OA, et al. DNA hydrolyzing autoantibodies. Science 1992; 256: 665-7.

21. Vlassov A, Florentz C, Helm $M$, et al. Characterization and selectivity of catalytic antibodies from human serum with RNase activity. Nucleic Acids Res 1998; 26 : 5243-50.

22. Baranovskii $A G$, Ershova NA, Buneva VN, et al. Catalytic heterogeneity of polyclonal DNA-hydrolyzing antibodies from the sera of patients with multiple sclerosis. Immunol Lett $2001 ; 76: 163-7$.

23. Lacroix-Desmazes S, Bayry J, Misra N, et al. The prevalence of proteolytic antibodies against factor VIII in hemophilia A. N Engl J Med 2002; 346: 662-7.

24. Wentworth JP, McDunn JE, Wentworth $A D$, et al. Evidence of antibody- catalyzed ozone formation in bacterial killing and inflammation. Science 2002; 298: 2195-9.

25. Wentworth $A$, Jones $L$, Wentworth $\mathrm{P}$, et al. Antibodies have the intrinsic capacity to destroy antigens. Proc Natl Acad Sci USA 2000; 97 10930-5.

26. Nardi M, Tomlinson S, Greco MA, Karpatkin S. Complement-independent, peroxide-induced antibody lysis of platelets in HIV-1related immune thrombocytopenia. Cell 2001; 106: 551-61.

27. Tawfik DS, Chap R, Green BS, et al. Unexpectedly high occurence of catalytic antibodies in MLR/Ipr and $\mathrm{SLJ}$ mice immunized with a transition-state analog: is there a linkage to autoimmunity. Proc Natl Acad Sci USA 1995; 92 : 2145-9.

28. Thomas NR. Hapten design for the generation of catalytic antibodies. Appl Biochem Biotechnol 1994; 47: 345-72.

\section{Séquençage du génome humain : le chromosome 14, quatrième chromosome terminé}

Roland Heilig, Núria Fonknechten
Genoscope, Centre

National de Séquençage,

2, rue Gaston Crémieux,

CP 5706, 91057 Évry Cedex,

France.

heilig@genoscope.cns.fr fonk@genoscope.cns.fr

dies génétiques. Restait à accomplir une tâche de « finition », tout aussi capitale, visant à déterminer la séquence manquante entre les contigs, puis à assurer en tout point un niveau strict de qualité. Cette étape, déjà accom-

$>$ En juin 2000, un consortium public regroupant 20 laboratoires de six pays annonça le séquençage de $90 \%$ du génome humain* [1] sous forme d'un arrangement encore imparfait (working draft) de plus de 150000

\footnotetext{
* II s'agit en fait de la partie euchromatique (soit environ $90 \%$ du génome). L'hétérochromatine, représentée surtout au niveau des centromères et des télomères et quasiment dépourvue de gènes, est difficilement accessible aux techniques usuelles de séquençage, en raison d'une structure répétitive particulière et d'une homologie souvent élevée entre les centromères des différents chromosomes.
}

segments (contigs) d'une longueur médiane $^{\star \star}$ de près de 90000 paires de bases (pb) et positionnés entre eux, par chromosome, sur la base d'informations de proximité [2] $(\rightarrow)$. Cette première esquisse constituait une avancée considérable, notamment en servant de support à la recherche des gènes responsables de mala-

\footnotetext{
** La moitié de la séquence est assemblée en contigs d'une taille égale ou supérieure à la longueur médiane.
}

plie pour les trois plus petits chromosomes, 22, 21 puis 20 [3-5], devrait être complétée pour les suivants au cours de cette année. Disposer d'une séquence finie et fiable reste essentiel pour nombre d'analyses fines du génome, et en particulier pour une description détaillée des gènes, de leurs mécanismes d'action et de régulation.

Le Genoscope (Centre $(\rightarrow) \mathrm{m} / \mathrm{s}$ national de séquençage) a $\quad 2001, n^{\circ}$ 